Journal homepage http://revistas.unitru.edu.pe/index.php/sSMM

SELECCIONES MATEMÁTICAS
Universidad Nacional de Trujillo
ISSN: $2411-1783$ (Online)
Vol. 04(02): $202-210(2017)$

Teoremas de Estabilidad en un Modelo Matemático SI con Dinámica Vital

Estructurado por Sexo para el Estado Libre de Infección desarrollado mediante las

Ecuaciones Diferenciales Ordinarias y las Ecuaciones Diferenciales con Retardo aplicado a la Salud Pública del Perú

\title{
Stability Theorems for a Mathematical Model SI with Vital Dynamics Structured by Sex for the Infection Free Steady developed by the Ordinary Differential Equations and the Delay Differential Equations respectively
}

Neisser Pino Romero* ${ }_{\text {and }}$ Roxana López Cruz ${ }^{* *}$

DOI: http://dx.doi.org/10.17268/sel.mat.2017.02.07

\begin{abstract}
Resumen
En el presente trabajo de investigación, se analiza cualitativamente un Modelo Matemático SI con Dinámica vital Estrucurado por Sexo desarrollado mediante las ecuaciones diferenciales ordinarias (transmisión de contagio es instantáneo), y también desarrollado por las ecuaciones diferenciales con retardo (transmisión de contagio se da después de un cierto periodo de tiempo), donde se proponen teoremas de Estabilidad Local y Asintótica para el punto libre de infección de ambos modelos, respectivamente. Los modelos permiten una concepción matemática de la dinámica de la enfermedad, y permitirían realizar una mejor previsión a las instituciones nacionales, de manera particular a la Dirección General de Epidemiología debido que es el encargado de realizar las actividades de previsión y de control de la enfermedad que serán considerados como políticas públicas por el Ministerio de Salud.
\end{abstract}

Palabras clave. Epidemiología Matemática. Ecuaciones Diferenciales Ordinarias. Ecuaciones Diferenciales con Retardo. Puntos Estacionarios. Estabilidad Local. Estabilidad Asintótica.

Abstract

In the present work, a Basic Model SI with Vital Dynamics Structured by Gender developed by the Ordinary Differential Equations (Transmission of contagion is instantaneous), and also developed in the Delay Differential Equations (Transmission of contagion occurs after a certain period of time), where the Local and Asymptotic Stability Theorem is proposed The Free of Infection point for both models, respectively.

Keywords. Mathematical Epidemiology. Ordinary Differential Equations. Delay Differential Equations. Stationary Points. Local stability. Asintotic Estability.

1. Introducción. El presente modelo que se desarrollará en E.D.O. es una perturbación inicial del Modelo de W. O. Kermack y A. G. McKendrick (1927), que en su tiempo permitió encontrar senderos en la investigación epidemiológica [10]. Después se propusó una nueva perturbación extendiendo el modelo para considerar las poblaciones estructuradas por sexo [7], con lo cual brindaba una percepción más amplia del

\footnotetext{
*Universidad Peruana Cayetano Heredia, Facultad de Ciencias y Filosofía Alberto Cazorla Talleri. UNMSM, Facultad de Ciencias Matemáticas, (E-mail:neisser.pino@upch.pe, neisser.pino@unmsm.edu.pe).

**UNMSM, Facultad de Ciencias Matemáticas, (E-mail:rlopezc@unmsm.edu.pe)
} 
problema epidemiológico por lo cual es necesario analizarlo matemáticamente. Luego, se realizó el análisis de la existencia y unicidad de las soluciones con su respectivo teorema de estabilidad local en [8,11].

En el presente trabajo, se presentará el Teorema de Estabilidad Local para el Modelo mediante las Ecuaciones Diferenciales Ordinarias, para después presentar y demostrar el Teorema de Estabilidad Absoluta para el Modelo mediante las Ecuaciones Diferenciales con Retardo. La relación entres estos dos teoremas consistirá que poseen la misma hipótesis, pero nos brindará la estabilidad que corresponde a cada sistema desarrollado. Donde epidemiológicamente el sistema en E.D.O. representa la dinámica de transmisión de una enfermedad infecciosa, donde se considerará que después del contagio se puede transmitir instantáneamente la enfermedad. [7,8].

Muchas de las enfermedades infecciosas poseen un periodo de incubación, o también llamado periodo latente, antes de poder transmitir la enfermedad, por lo cual se estudia un Sistema en E.D.R., que mediante el coeficiente de retardo representa muy bien el periodo latente de la enfermedad. En el Modelo representado mediante E.D.O., se considera el ingreso constante de individuos no susceptibles (población que no ha iniciado su vida sexual activa) a la población susceptible correspondiente. Se considera la salida de la población susceptible mediante la tasa de mortalidad natural para cada población de Infectados (Hombres y Mujeres), y a la vez también se considera la salida de la población de los infectados mediante la tasa de mortalidad tanto por la epidemia como la natural para cada población de Infectados (Hombres y Mujeres). $[1,2,3,4,5,8,9,10,11]$.

2. Modelo SI con Dinámica Vital en E.D.O.. En esta sección, nos centraremos en detallar brevemente los Resultados obtenidos de la Tesis de Licenciatura: "Modelo Matemático de la Dinámica de Transmisión del VIH/SIDA en una Población Heterosexual Activa en el Perú", que describe la problemática de la Epidemia del VIH/SIDA. [7].

El Modelo Matemático SI con Dinámica Vital Estructurado por Sexo será desarrollado mediante las E.D.O., donde se representará como un sistema de cuatro ecuaciones. Se considerará las poblaciones Epidemiológicas (Susceptibles e Infectados) y ambas Estructuradas por el sexo (Hombres y Mujeres). [6,7,8].

Consideraremos la interacción entre las poblaciones sexualmente activas, únicamente heterosexual. El contagio se realizará mediante la vía sexual ( $97 \%$ de contagio por este medio). También se incluirá las tasas de mortalidad natural y por la enfermedad para cada población Infectada. El ingreso de la población No susceptible (individuos que no se inician sexualmente) permitirá el aumento de la población Susceptible lo que generalmente sucede dentro de la sociedad. $[6,7,8]$.

Consideraremos las Tasas de Supervivencia Epidemiológicas:

$\begin{array}{llll}* & R_{S}^{H}=\frac{\sigma_{h} H_{N S}}{\mu} & : & \text { Tasa de Supervivencia de Susceptibles Hombres. } \\ * & R_{S}^{M}=\frac{\sigma_{m} M_{N S}}{\mu} \quad: & \text { Tasa de Supervivencia de Susceptibles Mujeres. } \\ * & R_{I}^{H}=\frac{\beta_{h}}{\alpha+\mu} & : & \text { Tasa de Supervivencia de Infectados Hombres. } \\ * & R_{I}^{M}=\frac{\beta_{m}}{\varepsilon+\mu} & : & \text { Tasa de Supervivencia de Infectadas Mujeres. }\end{array}$

En la siguiente Tabla donde se describe las Variables y los parámetros de nuestro Modelo. Y, las dinámicas que se tendrán en cuenta en el modelo son la esencia del modelo debido que intervienen la interacción entre variables que representarán los supuestos ya mencionados. [7,8]. 


\begin{tabular}{|c|l|c|}
\hline Variables & \multicolumn{1}{|c|}{ Descripción } & Valor \\
\hline$S_{h}^{\prime}$ & Cantidad de Susceptibles Hombres por unidad de Tiempo & \\
\hline$I_{h}^{\prime}$ & Cantidad de Infectados Hombres por unidad de Tiempo & \\
\hline$H_{N S}$ & Cantidad de Individuos Hombres NO Susceptibles & 2,0 (Miles) \\
\hline$S_{h}$ & Cantidad de Individuos Susceptibles Hombres & 1,0 (Miles) \\
\hline$I_{h}$ & Cantidad de Individuos Infectados Hombres & $0,2$ (Miles $)$ \\
\hline$S_{m}^{\prime}$ & Cantidad de Susceptibles Hombres por unidad de Tiempo & \\
\hline$I_{m}^{\prime}$ & Cantidad de Infectados Hombres por unidad de Tiempo & $2,0($ Miles $)$ \\
\hline$M_{N S}$ & Cantidad de Individuos Mujeres NO Susceptibles & $1,2($ Miles $)$ \\
\hline$S_{m}$ & Cantidad de Individuos Susceptibles Mujeres & $0,2($ Miles $)$ \\
\hline$I_{m}$ & Cantidad de Individuos Infectados Mujeres & Valor $(\%)$ \\
\hline Parámetros & Descripción & 0,015 \\
\hline$\sigma_{h}$ & Tasa de ingreso de los Hombres por unidad de Tiempo & 0,010 \\
\hline$\sigma_{m}$ & Tasa de ingreso de las Mujeres por unidad de Tiempo & 0,20 \\
\hline$\beta_{h}$ & $\begin{array}{l}\text { Tasa de contagio de una Mujer Susceptible por un Hombre } \\
\text { Infectado por unidad de Tiempo }\end{array}$ & 0,25 \\
\hline$\beta_{m}$ & $\begin{array}{l}\text { Tasa de contagio de un Hombre Susceptible por una Mujer } \\
\text { Infectada por unidad de Tiempo }\end{array}$ & 0,04 \\
\hline$\mu$ & Tasa de mortalidad natural por unidad de Tiempo & 0,03 \\
\hline$\alpha$ & $\begin{array}{l}\text { Tasa de mortalidad por la epidemia en los Hombres Infectados } \\
\text { por unidad de Tiempo }\end{array}$ & 0,03 \\
\hline$\varepsilon$ & $\begin{array}{l}\text { Tasa de mortalidad por la epidemia en las Mujeres Infectadas } \\
\text { por unidad de Tiempo }\end{array}$ & 5 \\
\hline & Coeficiente de Retardo Constante (Discreto) & \\
\hline
\end{tabular}

2.1. Modelo Matemático. El coeficiente de la Estrategia de Control $(\varpi)$ será un dato estadístico el cual determinará el indice de protección que se tiene, este coeficiente estará entre $[0,1]$. Por comodidad se utilizará el valor $w$, pues en sí, la estrategia de control será de la siguiente manera: $w=1-\varpi$. Donde $\varpi$ es el coeficiente de Efectividad de la Estrategia de Control. Esto mostrará que cuando no haya una estrategia de control $\varpi=0$, el valor de $w=1$; brindando un Modelo Matemático sin control, es decir, no se toma ninguna medida para intentar disminuir el contagio de la enfermedad, por ende si $\varpi=1$, el valor de $w=0$, lo que indicaría que la Estrategia de Control es completamente efectiva al $100 \%$. [7,8].

A continuación el sistema de ecuaciones que representará el modelo matemático mediante las Ecuaciones Diferenciales Ordinarias, para luego presentarlo de una manera similar mediante las Ecuaciones Diferenciales con Retardo.

$$
\begin{cases}S_{h}^{\prime} & =\sigma_{h} H_{N S}-w \beta_{m} I_{m} S_{h}-\mu S_{h} \\ I_{h}^{\prime} & =w \beta_{m} I_{m} S_{h}-\alpha I_{h}-\mu I_{h} \\ S_{m}^{\prime} & =\sigma_{m} M_{N S}-\beta_{h} I_{h} S_{m}-\mu S_{m} \\ I_{m}^{\prime} & =\beta_{h} I_{h} S_{m}-\varepsilon I_{m}-\mu I_{m} \\ S_{h}(0) & =S_{o}^{h} \geq 0 ; I_{h}(0)=I_{o}^{h} \geq 0 \\ S_{m}(0) & =S_{o}^{m} \geq 0 ; I_{m}(0)=I_{o}^{m} \geq 0 \\ 0<\alpha, \beta_{m}, \beta_{h}, \sigma_{h}, \sigma_{m}, \varepsilon, \mu, w<1\end{cases}
$$

2.2. Estado de Equilibrio del Modelo. Para la ecuación (1), consideraremos que existe y tiene solución única [7]. Por lo cual, ahora garantizaremos la estabilidad del modelo matemático, en primer lugar se hallará los puntos críticos del Modelo matemático, representado por un sistema de ecuaciones igualados a cero, y desarrollando el sistema obtendremos el Punto Libre de Infección y el Punto Endémico.

En nuestro caso, nos centraremos en el Punto Libre de Infección.

$$
\bar{E}=\left(\frac{\sigma_{h} H_{N S}}{\mu} ; 0 ; \frac{\sigma_{m} M_{N S}}{\mu} ; 0\right)
$$


2.2.1. Matriz de la Siguiente Generación. El método de la Siguiente Generacion es un método muy útil para determinar el Número Básico de Reproducción Efectiva $\Re_{e}$ para los Modelos Comportamentales. Este método nos permite determinar bajo qué condiciones el Sistema es Estable o Inestable Localmente. $[5,7,9]$.

Como la expresión (1) es un sistema no lineal de Ecuaciones Diferenciales Ordinarias, en primer lugar se va a linealizar el sistema. Para lo cual el Jacobiano del Sistema sería:

$$
\begin{aligned}
\text { Sea } X=\left(S_{h}, I_{h}, S_{m}, I_{m}\right) & \\
J(X) & =\left[\begin{array}{cccc}
-w \beta_{m} I_{m}-\mu & 0 & 0 & -w \beta_{m} S_{h} \\
w \beta_{m} I_{m} & -\alpha-\mu & 0 & w \beta_{m} S_{h} \\
0 & -\beta_{h} I_{m} & -\beta_{h} I_{h}-\mu & 0 \\
0 & \beta_{h} I_{m} & \beta_{h} I_{h} & -\varepsilon-\mu
\end{array}\right]
\end{aligned}
$$

Mediante el Método de la Matriz de la Siguiente Generación, la matriz Jacobiana se expresará de la siguiente manera: $J=T+\Sigma$.

$$
\begin{gathered}
T=\left[\begin{array}{cccc}
-w \beta_{m} I_{m} & 0 & 0 & -w \beta_{m} S_{h} \\
w \beta_{m} I_{m} & 0 & 0 & w \beta_{m} S_{h} \\
0 & -\beta_{h} S_{m} & -\beta_{h} I_{h}-\mu & 0 \\
0 & \beta_{h} S_{m} & \beta_{h} I_{h} & 0
\end{array}\right] \\
\Sigma=\left[\begin{array}{cccc}
-\mu & 0 & 0 & 0 \\
0 & -\alpha-\mu & 0 & 0 \\
0 & 0 & -\mu & 0 \\
0 & 0 & 0 & -\varepsilon-\mu
\end{array}\right]
\end{gathered}
$$

luego, $\quad K=T \cdot \Sigma^{-1}$

$$
K=\left[\begin{array}{cccc}
-\frac{w \beta_{m} Y_{m}}{\mu} & 0 & 0 & -\frac{w \beta_{m} X_{h}}{\varepsilon+\mu} \\
\frac{w \beta_{m} Y_{m}}{\mu} & 0 & 0 & \frac{w \beta_{m} X_{h}}{\varepsilon+\mu} \\
0 & -\frac{\beta_{h} X_{m}}{\alpha+\mu} & -\frac{\beta_{h} Y_{h}}{\mu} & 0 \\
0 & \frac{\beta_{h} X_{m}}{\alpha+\mu} & \frac{\beta_{h} Y_{h}}{\mu} & 0
\end{array}\right]
$$

Como $|K|=0$, no se podrá analizar mediante NGM con dominio grande, sino se reformulará mediante NGM con dominio pequeño de la siguiente manera:

$$
C=\left[\begin{array}{ll}
-1 & 0 \\
1 & 0 \\
0 & -1 \\
0 & 1
\end{array}\right] \quad R=\left[\begin{array}{llll}
w \beta_{m} Y_{m} & 0 & 0 & w \beta_{m} X_{h} \\
0 & \beta_{h} X_{m} & \beta_{h} Y_{h} & 0
\end{array}\right]
$$

así, $\quad K_{S}=-R \cdot \Sigma^{-1} \cdot C$

$$
K_{S}=\left[\begin{array}{cc}
-\frac{w \beta_{m} Y_{m}}{\mu} & \frac{w \beta_{m} X_{h}}{\varepsilon+\mu} \\
\frac{\beta_{h} X_{m}^{\mu}}{\alpha+\mu} & -\frac{\beta_{h} Y_{h}}{\mu}
\end{array}\right]
$$

Se buscará garantizar que $\left|K_{S}\right| \neq 0$, en caso que, $\left|K_{S}\right|=0$, no se podría utilizar el Método de la Matriz de la Siguiente Generación. 
Teorema 2.1 Si $\frac{R_{1}^{2}}{\mu^{2} w U_{4}} \neq \frac{(\varepsilon+\mu)(\alpha+\mu) U_{4}^{2}}{\beta_{h} \beta_{m} R_{2}}$, entonces $\left|K_{S}\right| \neq 0$. (Ver Demostración en [7]).

Luego, se puede hallar el Número Básico de Reproducción Efectiva:

$$
\Re_{e}=\sqrt{\left(\frac{w \beta_{m} I_{m}+\beta_{h} I_{h}}{2 \mu}\right)^{2}-\left(\frac{w \beta_{m} \beta_{h} I_{m} I_{h}}{\mu^{2}}-\frac{w \beta_{m} \beta_{h} S_{m} S_{h}}{(\varepsilon+\mu)(\alpha+\mu)}\right)}-\frac{w \beta_{m} I_{m}+\beta_{h} I_{h}}{2 \mu}
$$

Ahora, teniendo el valor de $\Re_{e}$, lo analizaremos en el Punto Libre de Infección $\bar{E}$.

$$
\Re_{e}=\sqrt{w \cdot R_{S}^{H} \cdot R_{S}^{M} \cdot R_{I}^{H} \cdot R_{I}^{M}}
$$

Teorema 2.2 Sea $w, R_{S}^{H}, R_{S}^{M}, R_{I}^{H}, R_{I}^{M}>0$. (Ver Demostración en [7]).

Si $w \cdot R_{S}^{H} \cdot R_{S}^{M} \cdot R_{I}^{H} \cdot R_{I}^{M}<1$, entonces $\Re_{e}<1$, por ende el Sistema (1) es Estable localmente para el Punto Libre de Infección $\bar{E}=\left(\frac{\sigma_{h} H_{N S}}{\mu} ; 0 ; \frac{\sigma_{m} M_{N S}}{\mu} ; 0\right)$.

3. Modelo SI con Dinámica Vital en E.D.R.. En muchas epidemias no ocurre una infección instántanea, sino el individuo pasa un tiempo para que pueda propagar la enfermedad. Bajo esta consideración, el Retardo nos permite considerar el tiempo en que se encuentra un individuo en la transición para que sea considerado infectado y que pueda transmitir la enfermedad, más aún, al considerar las poblaciones Estructuradas por Sexo nos permitirá tener una mejor comprensión de la dinámica que sucede mediante la interacción entre los individuos. Por lo cual, se introducirá el Retardo en la población Infectada femenina, y en la población susceptible masculina cuando haya una interacción entre ambas, teniendo una consideración para el Retardo $(\tau>0)$, debido que la Población de los Hombres ha ido en una tendencia de aumento en su vida sexual activa, por lo cual esta consideración nos permite entender que los Hombres tienen mucho más probabilidad de contraer la enfermedad. Por otra parte, la Población de las Mujeres tanto Susceptibles e Infectadas se considerarán como ecuaciones diferenciales ordinarias, es decir, no se le aplicará ningún Retardo por lo cual se interpretará que se mantiene en la misma dinámica expuesta anteriormente en la sección 3.1. [2,3,8,9,10,11].

3.1. Modelo Matemático. El Modelo Matemático considerará un coeficiente de Estrategia de Control, del mismo modo que el Modelo anterior, con el coeficiente de efectividad: $w=1-\varpi$. Donde $\varpi$ es representa la Estrategia de Control. [7].

$$
\left\{\begin{aligned}
& S_{h}^{\prime}=\sigma_{h} H_{N S}-w \beta_{m} \cdot S_{h} \cdot I_{m}-\mu S_{h} \\
& I_{h}^{\prime}=w \beta_{m} \cdot S_{h}(t-\tau) \cdot I_{m}(t-\tau)-(\alpha+\mu) I_{h} \\
& S_{m}^{\prime}=\sigma_{m} M_{N S}-\beta_{h} \cdot S_{m} \cdot I_{h}-\mu S_{m} \\
& I_{m}^{\prime}=\beta_{h} \cdot S_{m} \cdot I_{h}-(\varepsilon+\mu) I_{m} \\
& S_{h}(0)= S_{o}^{h}(t), \quad I_{h}(0)=I_{o}^{h}(t):[-\tau, 0] \longrightarrow[0, \infty+\rangle \\
& S_{m}(0)= S_{o}^{m}(t), \quad I_{m}(0)=I_{o}^{m}(t) \\
& \sigma_{h}, \sigma_{m}, w, \beta_{h}, \beta_{m}, \alpha, \mu, \varepsilon>0
\end{aligned}\right.
$$

3.2. Estado de Equilibrio del Modelo. Realizaremos el análisis cualitativo para el Modelo Matemático SI correspondiente al sistema (2). Por lo cual tendremos que hallar, en primer lugar sus puntos críticos asociados al sistema. Una consideración bastante importante, es que los puntos críticos del sistema (2) serán los mismos que en el sistema (1).

$$
\widetilde{E_{1}}=\left(\frac{\sigma_{h} H_{N S}}{\mu} ; 0 ; \frac{\sigma_{m} M_{N S}}{\mu} ; 0\right)
$$


3.2.1. Linelización del Sistema. Del mismo modo que se hizo en las Ecuaciones Diferenciales Ordinarias con respecto al Sistema No Lineal, mediante una linealización del Sistema. Se realizará un análisis similar con la matriz Jacobiana con respecto a las variables sin Retardo, y una matriz Jacobiana con respecto a las variables con Retardo. [6,7,8,9].

Por lo cual la Matriz Jacobiana sin Retardo sería el siguiente:

$$
J(S, I)=\left[\begin{array}{cccc}
-\beta_{m} I_{m}-\mu & 0 & 0 & -\beta_{m} S_{h} \\
0 & -(\alpha+\mu) & 0 & 0 \\
0 & -\beta_{h} S_{m} & -\beta_{h} I_{h}-\mu & 0 \\
0 & \beta_{h} S_{m} & \beta_{h} I_{h} & -(\varepsilon+\mu)
\end{array}\right]
$$

Por lo cual la Matriz Jacobiana con Retardo sería el siguiente:

$$
J_{\tau}(S, I)=\left[\begin{array}{cccc}
0 & 0 & 0 & 0 \\
\beta_{m} \cdot w \cdot I_{m}(t-\tau) & 0 & 0 & \beta_{m} \cdot w \cdot S_{h}(t-\tau) \\
0 & 0 & 0 & 0 \\
0 & 0 & 0 & 0
\end{array}\right]
$$

Vemos que el determinante de $J_{\tau}$ es nula, este detalle lo consideraremos más adelante. Teniendo las matrices Jacobianas, formaremos el polinomio característico que tiene la forma:

$$
p(\lambda)=\operatorname{det}\left(\lambda \cdot I d-J-J_{\tau} \cdot e^{-\lambda \tau}\right)=0
$$

3.2.2. Punto Libre de Infección. El punto libre de infección $\widetilde{E_{1}}=\left(\frac{\sigma_{h} H_{N S}}{\mu} ; 0 ; \frac{\sigma_{m} M_{N S}}{\mu} ; 0\right)$ representa cuando en un instante del tiempo ya no hay infectados, solamente hay susceptibles. Analizaremos este punto de equilibrio para saber como es su comportamiento a través del tiempo.

Sea la consideración: $d_{1}=\alpha+\mu, d_{2}=\varepsilon+\mu$.

Luego, el polinomio característico para El Punto Libre de Infección, $\widetilde{E_{1}}$

$$
p(\lambda)=(\lambda+\mu)^{2}\left(\lambda^{2}+\left(d_{1}+d_{2}\right) \lambda+d_{1} d_{2}-w\left(\frac{\beta_{h} \sigma_{h} H_{N S}}{\mu}\right)\left(\frac{\beta_{m} \sigma_{m} M_{N S}}{\mu}\right) \cdot e^{-\lambda \tau}\right)
$$

así, se puede deducir

$$
\lambda_{1}=-\mu<0
$$

ahora, el siguiente autovalor debería ser negativo o tener parte real negativa para poder concluir que es estable el punto libre de infección.

Sean los polinomios,

$$
\begin{gathered}
\bar{p}(\lambda)=\lambda^{2}+\left(d_{1}+d_{2}\right) \lambda+d_{1} d_{2} \\
\bar{q}(\lambda)=-w\left(\frac{\beta_{h} \sigma_{h} H_{N S}}{\mu}\right)\left(\frac{\beta_{m} \sigma_{m} M_{N S}}{\mu}\right)
\end{gathered}
$$

así, el polinomio característico tiene la forma

$$
p(\lambda)=\bar{p}(\lambda)-\bar{q}(\lambda) \cdot e^{-\lambda \tau}
$$

Proposición 4.1 Sean $p, q$ polinomios con coeficientes reales. Supongamos lo siguiente: 
1. $p(\lambda) \neq 0, \Re(\lambda)>0$

2. $|q(i y)|<|p(i y)|, 0 \leq y<\infty+$

3. $\lim _{|\lambda| \longrightarrow \infty+, \Re(\lambda) \geq 0}\left|\frac{q(\lambda)}{p(\lambda)}\right|=0$

Entonces, $\Re(\lambda)<0$ para toda raíz $\lambda$, y $r \geq 0$ del polinomio característico (3). (Ver [8,10]).

La conclusión de la Proposición 4.1 es llamado Estabilidad Absoluta, donde la estabilidad se mantiene para todo valor del Retardo. [10].

Corolario 4.1 Sea $p$ un polinomio con coeficientes reales, y tiene el coeficiente uno en el término de mayor orden. Y también, sea $q=c$ una constante.

Si todas las raíces de $p$ son reales y tienen parte negativa, y $|p(0)|>|c|$. Entonces, $\Re(\lambda)<0$, para toda raíz $\lambda$, y $r \geq 0$. (Ver [10]).

Teniendo la consideración de $\left|J_{\tau}\right|=0$. Analizaremos la estabilidad local del punto Libre de Infección, por lo cual ahora utilizaremos los resultados de la Proposición 4.1, en particular el Corolario 4.1, debido a una consideración que el polinomio $\bar{q}(\lambda)$ es una constante. Con este Corolario conseguiremos establecer la Estabilidad Absoluta. [10].

Tenemos: $\bar{p}(\lambda)=\lambda^{2}+\left(d_{1}+d_{2}\right) \lambda+d_{1} d_{2} \quad y \quad \bar{q}(\lambda)=-\frac{\beta_{h} \sigma_{h} H_{N S} \beta_{m} \sigma_{m} M_{N S}}{\mu^{2}} \cdot e^{-w \tau}$

La discriminante de $\bar{p}(\lambda):\left(d_{1}+d_{2}\right)^{2}-4 d_{1} d_{2}=\left(d_{1}-d_{2}\right)^{2} \geq 0$

Las raíces de $\bar{p}(\lambda)$ :

$$
\lambda_{1}=-d_{2}<0 \quad y \quad \lambda_{2}=-d_{1}<0
$$

Por consiguiente la primera condición está demostrada. Pasaremos a la segunda condición.

$$
(\alpha+\mu)(\varepsilon+\mu)>\left|-\frac{\beta_{h} \sigma_{h} H_{N S} \beta_{m} \sigma_{m} M_{N S}}{\mu^{2}} \cdot e^{-\varpi \tau}\right|
$$

Luego

$$
(\alpha+\mu)(\varepsilon+\mu)>\frac{\beta_{h} \sigma_{h} H_{N S} \beta_{m} \sigma_{m} M_{N S}}{\mu^{2}} \cdot e^{-\varpi \tau}
$$

La expresión anterior podemos expresarlo de la siguiente manera:

$$
w\left(\frac{\sigma_{h} H_{N S}}{\mu}\right)\left(\frac{\sigma_{m} M_{N S}}{\mu}\right)\left(\frac{\beta_{h}}{\alpha+\mu}\right)\left(\frac{\beta_{m}}{\varepsilon+\mu}\right)<1
$$

Por consiguiente:

$$
w \cdot R_{S}^{H} \cdot R_{S}^{M} \cdot R_{I}^{H} \cdot R_{I}^{M}<1
$$

Teorema 3.1 Sea $w, R_{S}^{H}, R_{S}^{M}, R_{I}^{H}, R_{I}^{M}>0$. ( Ver Demostración en [10]) Si $w \cdot R_{S}^{H} \cdot R_{S}^{M} \cdot R_{I}^{H} \cdot R_{I}^{M}<1$, entonces el Sistema (2) posee la Estabilidad Absoluta para el Punto Libre de Infección $\widetilde{E_{1}}=\left(\frac{\sigma_{h} H_{N S}}{\mu} ; 0 ; \frac{\sigma_{m} M_{N S}}{\mu} ; 0\right)$. .

4. Simulación Computacional. Las Simulaciones computacionales nos permiten visualizar el comportamiento que realiza las soluciones, mediante los Métodos Numéricos que nos brinda soluciones aproximadas. Por lo cual realizaremos las simulaciones computacionales de cada sistema de ecuaciones, es decir, tanto para las Ecuaciones Diferenciales Ordinarias como las de Retardo. Por lo cual, se utilizará los valores expresados en la Tabla 1 de la sección 3. Donde la gran diferencia lo brindará el coeficiente de Retardo, $\tau$. Este coeficiente mostrará que a base de la obtención de datos de las poblaciones epidemiológicas se puede tener una mejor visión de la epidemia y su manera más acertada de intervención para el bienestar de la sociedad. 

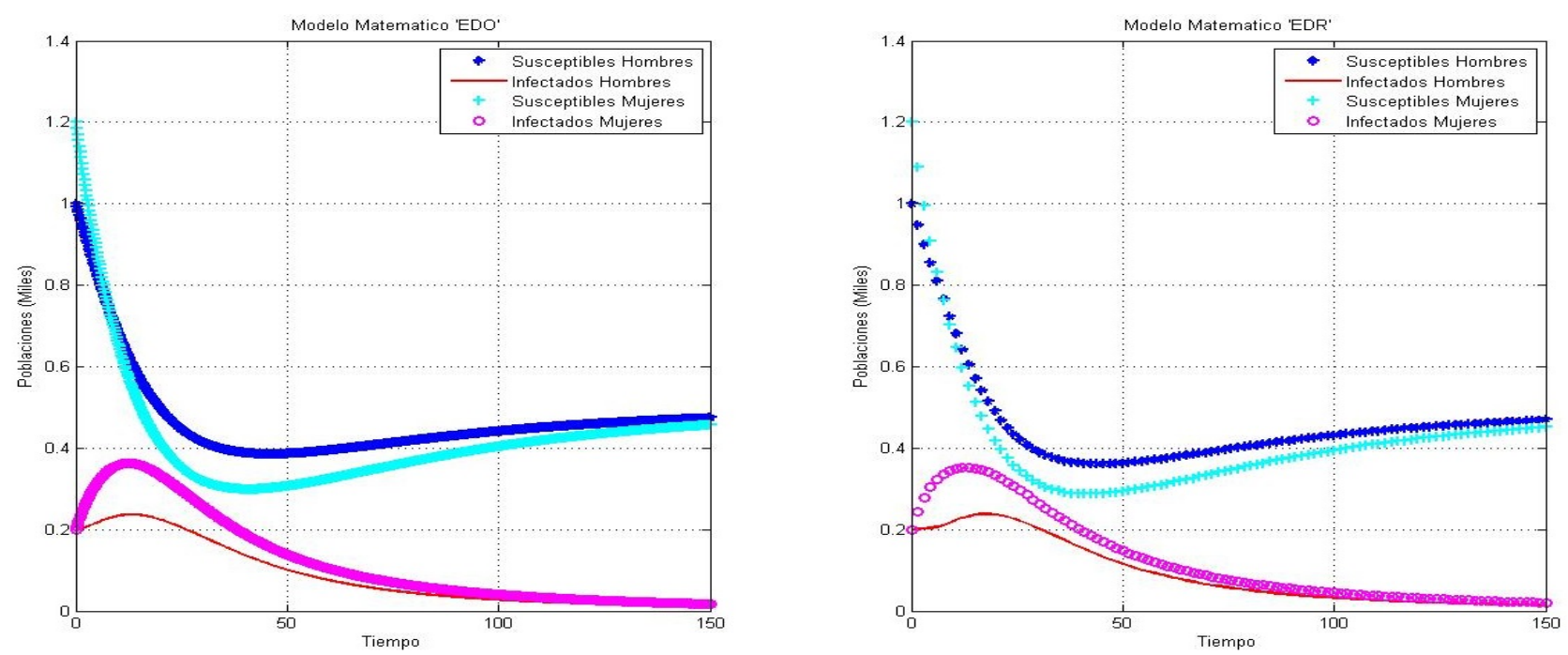

De la Simulación computacional, realizada con MATLAB, se puede analizar el comportamiento de las soluciones a través del tiempo considerado mediante el método de Runge Kutta de orden 4 para las E.D.O. y el método de Multipasos para las E.D.R. Epidemiológicamente se puede observar como se desarrolla la dinámica de transmisión de cada población, en especial la población infectada tanto de Hombres como de Mujeres. El sistema en E.D.R. nos permite saber que hay una leve constancia de la población Infectada de Hombres debido que es donde se ha aplicado el retardo, considerando la historia que se tiene. El coeficiente de retardo representa la cantidad de tiempo que se considerará dentro de la Historia que tiene de la población infectada para poder propagar la enfermedad. Estos resultados numéricos nos permiten complementar los resultados análiticos presentados mediante los teoremas de estabilidad.

5. Conclusiones. Los Modelos que se han presentado en las Secciones 3 y 4, tanto por las Ecuaciones Diferenciales Ordinarias como por las Ecuaciones Diferenciales con Retardo, respectivamente nos permiten realizar la principal observasión a considerar que ambos Teoremas enunciados poseen la misma condición, pero nos brindan diferentes resultados sobre la Estabilidad de cada sistema para el Punto Estacionario de Libre de Infección.

Como se conoce sobre la dificultad de los Sistemas No Lineales para poder hallar las soluciones análiticas, en lo general, se analiza el comportamiento de las soluciones (análisis cualitativo) y se complementa con simulaciones computacionales (análisis numérico); para poder obtener resultados que permitan inferir situaciones favorables o en qué caso desfavorables del problema epidemiológico. [1,5,9,10].

Por lo cual, podemos obtener las siguientes conclusiones.

1. Matemáticamente, el Sistema (1) mediante las E.D.O. y el Sistema (2) mediante las E.D.R. poseen la Estabilidad Local y la Estabilidad Absoluta para el Punto Libre de Infección, respectivamente mediante el Teorema 3.2 y el Teorema 4.1 presentados y demostrados.

2. Matemáticamente, el Teorema 3.2 y el Teorema 4.1 comparten la misma condición para garantizar sus respectivos resultados de Estabilidad.

3. Epidemiológicamente, el Modelo matemático que describe la dinámica de transmisión de una enfermedad infecciosa, de manera particular el VIH/SIDA, llega a estabilizarse en el momento que la población Infectada desciende considerablemente por mientras que la población Susceptible se va manteniendo en una población constante, esto describe el Punto Libre de Infección en el estado estacionario estudiado.

4. Ambos sistemas nos permite modelar la dinámica de transmisión de una enfermedad infecciosa (Susceptible-Infectado), bajo las perspectivas que se esté considerando intervenir dentro de los margenes de la Salud Pública. Por lo cual el modelamiento en E.D.O. siempre nos permite conocer el comportamiento cuando el contagio es inmediato, por mientras que el modelamiento E.D.R. nos permite conocer cuando se considere el periodo latente adecuado. 


\section{REFERENCIAS BIBLIOGRÁFICAS}

[1] Barrios Ginart J., Marrero Severo A., Baguer Díaz-Romañach M., De Arazoza Rodríguez H. (2010). Estimación de parámetros en modelos epidemiológicos deVIH/SIDA. Revista de Matemática: Teoría y aplicaciones. CIMPA - UCR.

[2] Erwin Forde Jonathan. Delay Differential Equation Model in Mathematical Biology. The University of Michigan, (2005).

[3] Driver Rodney David. Ordinary and Delay Differential Equations. Mathl. Comput. Modelling Vol. 24, No. 9, pp. 63-68, (1996).

[4] Gourley. S. A. Y KuAng Yang. A Stage Structured Predator-Prey Model and its dependence on Maturation Delay and Death Rate. J. Math. Biol., 49:188-200 (2004).

[5] Kuang Yang. (2002). Basic Properties of Mathematical Population Models. Department of Mathematics and Statistics, Arizona State University.

[6] López Cruz R. (2006). Structured SI Epidemic Models with Applications to HIV Epidemic. Arizona State University. pp. 27-45.

[7] Mesa Mazo M, Vergao Salazar Juan, Sanchez Botero Claudia, Muoz Loaiza Anibal. (2010). Modelo matemático para la dinámica de transmisión del VIH/SIDA en una población sexualmente activa. Universidad de Quindio, Armenio, Colombia. Rev. Salud Pública 12,308-316.

[8] Pino Romero N. (2013). Modelo matemático para la dinámica de transmisión del VIH/SIDA en una población heterosexual activa en el Perú. Facultad de Ciencias Matemáticas, UNMSM.

[9] Pino Romero N. (2017). Análisis y Simulación Numérica de un Modelo Matemático SI con Retardo Discreto para las Enfermedades de Transmisión Sexual. Facultad de Ciencias Matemáticas, UNMSM.

[10] Smith, H. (2010). An introduction to delay differential equations with applications to the life sciences (Vol. 57). Springer Science \& Business Media.

[11] W. O. KeRMACK \& A. G. MCKENDRICK. (1927). A contribution to the Mathematical theory of Epidemics Proceedings of the Royal Society of London Series A, 115:700-721. 